\title{
Editorial
}

\section{Applications of Methods of Numerical Linear Algebra in Engineering}

\author{
Masoud Hajarian, ${ }^{1}$ Feng Ding, ${ }^{2}$ and Jein-Shan Chen $^{3}$ \\ ${ }^{1}$ Department of Mathematics, Faculty of Mathematical Sciences, Shahid Beheshti University, General Campus, \\ Evin, Tehran 19839, Iran \\ ${ }^{2}$ School of Internet of Things Engineering, Jiangnan University, Wuxi 214122, China \\ ${ }^{3}$ Department of Mathematics, National Taiwan Normal University, Taipei 11677, Taiwan
}

Correspondence should be addressed to Masoud Hajarian; mhajarian@aut.ac.ir

Received 18 May 2014; Accepted 18 May 2014; Published 26 May 2014

Copyright ( 2014 Masoud Hajarian et al. This is an open access article distributed under the Creative Commons Attribution License, which permits unrestricted use, distribution, and reproduction in any medium, provided the original work is properly cited.

Methods of numerical linear algebra are concerned with the theory and practical aspects of computing solutions of mathematical problems in engineering such as image and signal processing, telecommunication, data mining, computational finance, bioinformatics, optimization, and partial differential equations. In recent years, applications of methods of numerical linear algebra in engineering have received a lot of attention and a large number of papers have proposed several methods for solving engineering problems. This special issue is devoted to publishing the latest and significant methods of numerical linear algebra for computing solutions of engineering problems.

We received twenty-five papers in the interdisciplinary research fields. This special issue includes ten high quality peer-reviewed articles.

In the following, we briefly review each of the papers that are published.

In the paper entitled "A model based on cocitation for web information retrieval," Y. Xie and T.-Z. Huang propose a new hyperlink weighting scheme to describe the strength of the relevancy between any two webpages.

In the paper entitled "Optimal grasping manipulation for multifingered robots using semismooth Newton method," C.-H. Ko and J.-S. Chen perform the optimal grasping control to find both optimal motion path of the object and minimum grasping forces in the manipulation.

In the paper entitled "(Anti-)Hermitian generalized (anti-) Hamiltonian solution to a system of matrix equations,"
J. Yu et al. propose the necessary and sufficient conditions for the existence of and the expression for the (anti-)Hermitian generalized (anti-)Hamiltonian solutions to the system of matrix equations $A X=B, C X=D$.

In the paper entitled "Dynamic model of a wind turbine for the electric energy generation," J. de J. Rubio et al. introduce a novel dynamic model for the modeling of the wind turbine behavior.

In the paper entitled "The extrapolation-accelerated multilevel aggregation method in PageRank computation," B.$\mathrm{Y}$. $\mathrm{Pu}$ et al. present an accelerated multilevel aggregation method for calculating the stationary probability vector of an irreducible stochastic matrix in PageRank computation.

In the paper entitled "A double-parameter GPMHSS method for a class of complex symmetric linear systems from Helmholtz equation," C.-X. Li and S.-L. Wu introduce a double-parameter GPMHSS (DGPMHSS) method for solving a class of complex symmetric linear systems from Helmholtz equation.

In the paper entitled "The explicit identities for spectral norms of circulant-type matrices involving binomial coefficients and harmonic numbers," J. Zhou et al. investigate the explicit formulae of spectral norms for circulant-type matrices.

In the paper entitled "Dynamic stability of Euler beams under axial unsteady wind force," Y.-Q. Huang et al. study the critical frequency equation for simply supported Euler beams with uniform section under arbitrary axial dynamic forces. 
In the paper entitled " $A$ modified conjugacy condition and related nonlinear conjugate gradient method," S. Yao et al. present a nonlinear conjugate gradient method that is globally convergent under the strong Wolfe Powell line search for general functions.

In the paper entitled "Spectral properties of the iteration matrix of the HSS method for saddle point problem," Q.-F. Cui et al. discuss spectral properties of the iteration matrix of the HSS method for saddle point problems.

\section{Acknowledgments}

The editors of this special issue would like to express their gratitude to the authors who have submitted manuscripts for consideration. They also thank the many individuals who served as referees of the submitted manuscripts.

Masoud Hajarian

Feng Ding

Jein-Shan Chen 


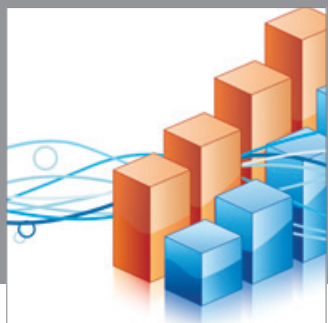

Advances in

Operations Research

mansans

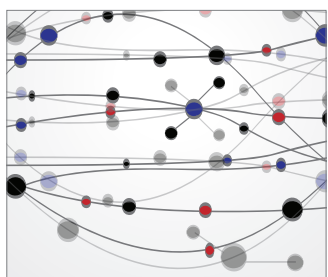

The Scientific World Journal
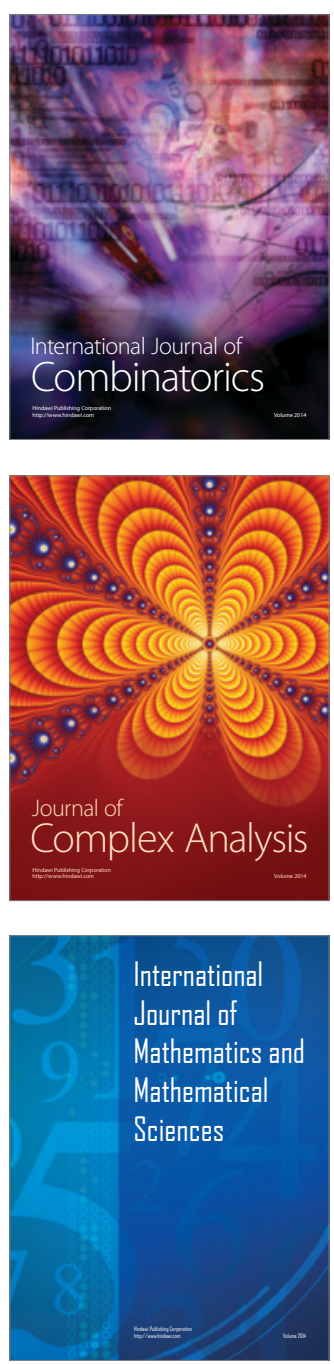
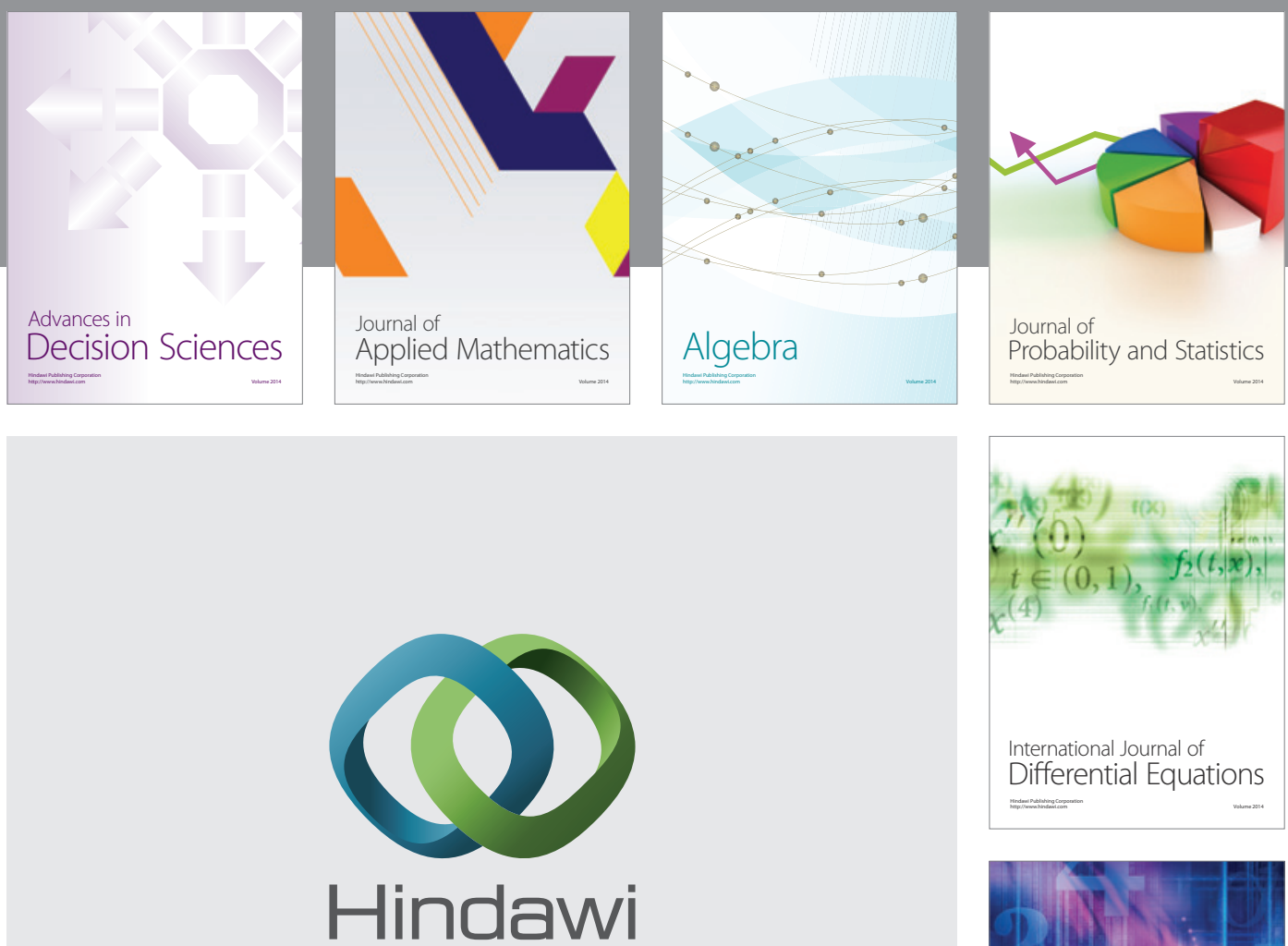

Submit your manuscripts at http://www.hindawi.com
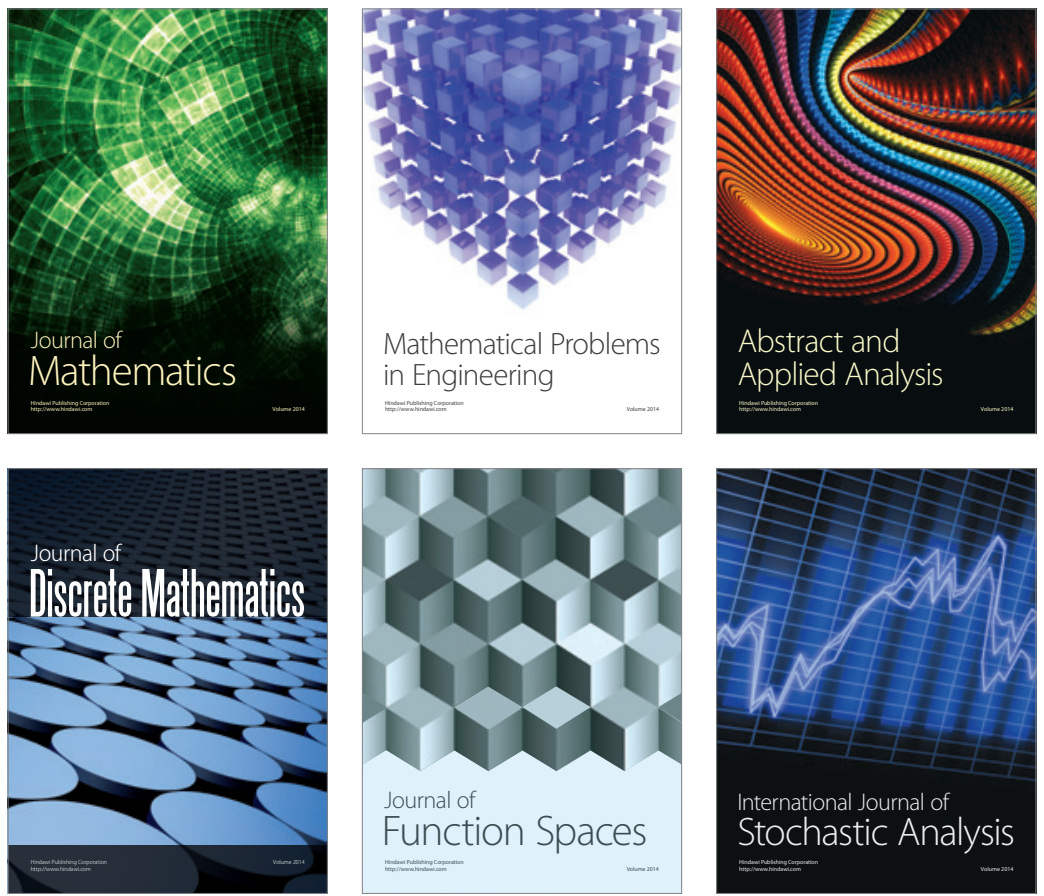

Journal of

Function Spaces

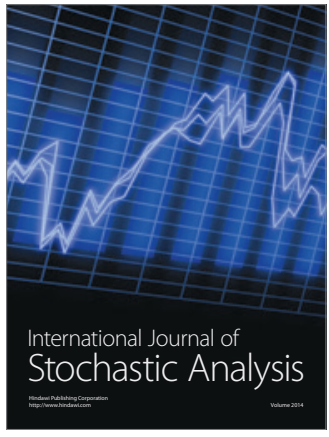

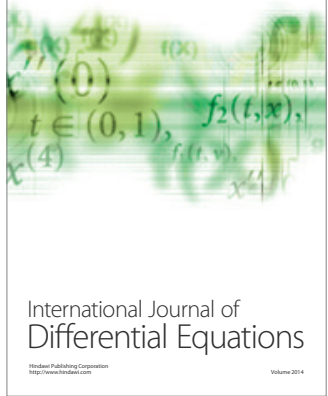
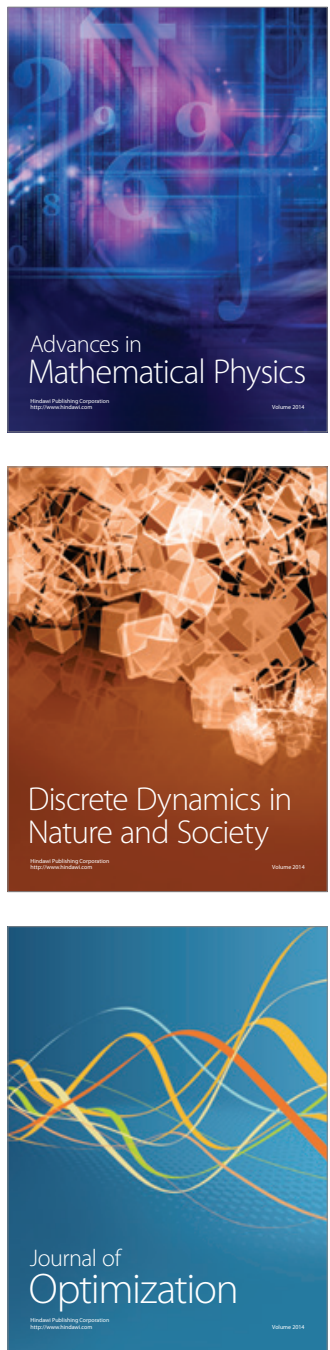